\title{
ALONGAMENTO ESTÁTICO E TREINAMENTO DE FORÇA: UMA BREVE REVISÃO DE LITERATURA
}

\section{Static stretching and strength training: a brief review of literature}

\author{
Gustavo Barquilha ${ }^{1,2}$ \\ ${ }^{1}$ Instituto de Ciências da Atividade Física e Esporte - Universidade Cruzeiro do Sul \\ ${ }^{2}$ Grupo de Estudos e Pesquisa em Exercício Físico e Fisiologia Aplicada - Unifieo
}

\begin{abstract}
Resumo: A busca pela maximização dos resultados obtidos com um protocolo de treinamento de força vem levando a uma discussão sobre uma possível interferência do alongamento antes de um treinamento de força. Nesse sentido, o objetivo deste estudo de revisão foi investigar a influência aguda do alongamento estático no desempenho em um protocolo de treinamento de força. Foram utilizadas como fontes as bases de dados PUBMED e SCIELO, assim como livros especializados no assunto. Na base de dados PUBMED foram utilizadas as palavras "stretching and strength", e na base de dados SCIELO as palavras-chave "alongamento e força". Foram encontrados, ao todo, 1026 artigos. Como critérios de inclusão foram escolhidos apenas artigos na língua inglesa ou portuguesa, publicados em um período de até 15 anos (entre 1994 e 2009), que utilizassem alongamentos estáticos em sua metodologia, além de um grupo controle e uma amostra com um numero de participantes compatíveis com o objetivo do trabalho. Considerados tais critérios, foram selecionados 31 artigos. Analisando a literatura escolhida, foram encontrados indícios de que o alongamento pode interferir no desempenho em um treinamento de força, principalmente quando referido alongamento é realizado em alto volume. Entretanto, a razão da existência dessa interferência ainda é um tema controverso, fazendo-se necessários novos estudos para elucidar a questão. Palavras Chave: Alongamento; Força; Desempenho.
\end{abstract}

\begin{abstract}
The search for the maximization of the results obtained with a strength training protocol has led to a discussion about a possible interference of stretching before strength training. In this sense, the objective of this review study was to investigate the influence of acute static stretching on performance in a strength training protocol. Were used as sources the databases PUBMED and SCIELO, as well as specialized books on the subject. The keywords searched were "stretching and strength" both writen in english at PUBMED and in portuguese at SCIELO. There were found 1026 articles. Inclusion criteria were selected only articles in English or Portuguese language, published in a period of up to 15 years (between 1994 and 2009), who used static stretches in its methodology, and a control group and one sample with a number of participants compatible with the objective of the work. Considered such criteria, we selected 31 items. When studying the selected literature, evidences that stretching can interfere on performance during a strength training were found, especially when stretching is performed at high volume. However, the reason for the existence of such interference is still a controversial issue, making necessary further studies to elucidate the issue.
\end{abstract}

Key Words: Stretching, Strengh, Performance.

\section{INTRODUÇÃO}

Estudos têm demonstrado que o treinamento de força pode provocar efeitos positivos em patologias como hipertensão( ${ }^{(1)}$, diabetes ${ }^{(2)}$, obesidade ${ }^{(3)}$, osteoporose $^{(4)}$, entre outras, além de proporcionar aumentos na força $a^{(5)}$ e potência muscular ${ }^{(6)}$, sendo essas duas últimas adaptações importantes também para atletas de alto rendimento. Para se obter melhores resultados com o treinamento, é necessária uma correta prescrição das variáveis envolvidas com o treinamento, como, por exemplo, o intervalo de descanso, intensidade e volume do treinamento, tipo de exercício, entre outros ${ }^{(7)}$.
Geralmente, o treinamento de força é realizado depois de um protocolo de exercícios de aquecimento, sendo freqüentemente utilizados exercícios de alongamento como forma de aquecimento antes do início da sessão. O aquecimento serve, em tese, para elevar a temperatura corporal, aumentar a demanda de oxigênio para o organismo através do aumento do fluxo sanguíneo, aumentar a lubrificação das articulações melhorando a viscosidade dos tendões, aumentar a velocidade das reações metabólicas, dentre outras adaptações ${ }^{(8)}$.

Entretanto, o alongamento pode acarretar alterações morfológicas no tecido conectivo e 
nas fibras musculares, com a ativação de órgãos sensoriais e microlesões, principalmente quando não estruturado corretamente ${ }^{(9,10)}$. Neste sentido, o alongamento deixa de ser apenas um exercício de aquecimento e passa a acarretar modificações importantes no organismo. Sendo assim, o objetivo deste estudo de revisão foi investigar a influência aguda do alongamento no desempenho em um protocolo de treinamento de força. Foram utilizadas como fontes as bases de dados PUBMED e SCIELO, assim como livros especializados no assunto. O presente estudo de revisão concentrase nos estudos que analisaram os efeitos agudos de sessões de alongamento realizadas antes de uma sessão de treinamento de força.

\section{REVISÃO DE LITERATURA}

\section{Influência do alongamento no desempenho em exercícios de força}

Os estudos sobre o tema alongamento antes da realização de um treinamento de força ainda são um tanto controversos. Diversos estudos têm demonstrado queda no rendimento quando se aplica um alongamento antes de um treinamento de força ${ }^{(11-13)}$. Outros, porém, não encontraram interferência do alongamento na produção de força ${ }^{(14,15)}$.

Arruda et $\mathrm{al}^{(16)}$ encontraram uma redução significante no desempenho em teste de 10 RM no exercício supino em um grupo que alongou antes dos testes, sendo que não foram encontradas diferenças estatísticas em um grupo que aqueceu no próprio aparelho antes de fazer os testes. Já Tricoli e Paulo(11) realizaram um estudo em que o objetivo foi investigar o efeito agudo dos exercícios de alongamento estático no desempenho de força máxima. Onze voluntários realizaram um teste de $1 \mathrm{RM}$ com e sem alongamento antes dos testes. Para o grupo que alongou, foram realizados seis exercícios com três repetições de 30 segundos cada. Já para o teste sem alongamento, o grupo realizou o teste de 1RM após umbreve aquecimento. Foram encontradas reduções significativas para o grupo que realizou alongamento antes do teste, tendo os autores concluído que uma sessão de alongamento pode reduzir o desempenho em um protocolo de força. Recentemente, Endlich et al ${ }^{(17)}$ realizaram um estudo com o intuito de analisar o efeito agudo do alongamento com diferentes tempos no desempenho da força dinâmica de membros superiores e inferiores em homens jovens. 14 voluntários participaram do estudo realizando um teste de $10 \mathrm{RM}$ em três situações distintas: condição sem alongamento, aquecimento específico seguido do teste de 10-RM; condição com oito minutos de alongamento (AL-8), uma sessão de alongamento estático com oito minutos de duração, seguido do aquecimento e teste de 10RM; e a condição alongamento 16 minutos (AL-16), 16 minutos de alongamento seguidos dos procedimentos descritos anteriormente. Os testes foram feitos no supino reto e leg-press $45^{\circ}$, e os alongamentos foram selecionados de forma a atingir as musculaturas solicitadas nos respectivos exercícios. Os autores encontraram redução da força muscular dinâmica de membros superiores e membros inferiores, tendo os autores concluído que sessões de alongamentos estáticos efetuados antes de atividades que envolvam força dinâmica possuem a capacidade de alterar negativamente o desempenho dessa qualidade física, acarretando pior rendimento em longos períodos de alongamento. Costa et al(18) realizaram um estudo que tinha como objetivo verificar a influência aguda do alongamento estático no desempenho de força máxima de 20 atletas de jiu-jitsu, tendo os autores concluído que o protocolo de alongamento utilizado gerou efeito deletério na geração de força máxima dos atletas avaliados. Também recentemente, Souza et al(19) verificaram o efeito agudo de intervalos passivos e do alongamento estático entre as séries sobre o número de repetições máximas (RM), percepção subjetiva de esforço (PSE) e volume total (VT) do número de repetições em um protocolo de séries múltiplas, com sobrecarga ajustada pelo teste de 8RM. Participaram deste estudo 14 homens divididos em duas situações experimentais: teste de 8RM com intervalo passivo e teste de 8RM com exercício de alongamento. Foram realizadas três séries no supino reto e no agachamento, tendo os autores concluído que o alongamento estático entre as séries também pode provocar diminuição no desempenho da força em 8RM.

Contrariando os resultados apresentados acima, Yamaguchi e Ishii ${ }^{(20)}$ realizaram um estudo em que onze voluntários realizaram três diferentes protocolos: uma sessão com cinco exercícios de alongamento estático e uma sessão 
sem alongamento, ambos antes do exercício leg press. Não foram encontradas reduções na produção de força com o grupo que realizou o alongamento estático e com o grupo que não

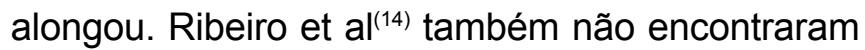
diferença estatisticamente significante no teste de $10 \mathrm{RM}$ realizado no exercício leg press. Foram realizados dois protocolos de exercícios, um com alongamento durante 30 segundos e outro com um aquecimento específico de 20 repetições leves antes de realizar o exercício selecionado. Provavelmente, estes resultados se devem ao baixo volume do alongamento realizado antes dos exercícios de força, indicando que exercícios de alongamento com baixo volume não interferem em exercícios de força. Sendo assim, um fator importante a ser considerado é o volume dos protocolos de treinamento de alongamento. Estudos têm demonstrado que quanto maior for o volume (tempo) de alongamento, maior a probabilidade de se encontrar uma influência negativa na produção de força após a realização desse alongamento ${ }^{(21,22)}$.

\section{Possíveis mecanismos envolvidos}

É controverso ainda o real mecanismo que leva a redução na produção de força decorrente do alongamento. Uma das possíveis causas da interferência do alongamento na produção de força pode ser o aumento da atividade do sistema parassimpático após a sua realização, ocasionando assim um relaxamento muscular ${ }^{(23)}$. Porém essa ativação do sistema parassimpático com a realização do alongamento parece ocorrer acima de 15 minutos de pratica de alongamento. Essa pode ser também a razão para alguns estudos com baixo volume de exercícios de alongamento não encontrarem interferência no desempenho em testes de exercícios resistidos.

Oalongamento pode desencadear mecanismos neurais que estimulam o órgão tendinoso de golgi e dos receptores de dor, o que pode desencadear a inibição de força ${ }^{(24,25)}$. Alguns autores citam que o alongamento possui a capacidade de modificar as propriedades viscoelásticas da unidade músculo-tendão, reduzindo assim a tensão e rigidez da unidade motora. É bom salientar que uma das funções dos tendões é a de transferir a força produzida pelo músculo esquelético para articulações e ossos, sendo que uma unidade músculo-tendão mais rígida pode transmitir de maneira mais eficiente as alterações de tensão no músculo(26,27). Outro fator além da redução na rigidez músculo-tendínea, são possíveis decréscimos na ativação das unidades motoras e da musculatura esquelética como um todo ${ }^{(24)}$ pode ser também um dos fatores responsáveis pela queda no desempenho de força após a realização de exercícios de alongamento.

Marek et $\mathrm{al}^{(28)}$ observaram uma diminuição da atividade elétrica do músculo após a realização de alongamento estático, ocorrendo assim uma diminuição da força e potência muscular. Esses achados corroboram um estudo de revisão publicado por Shrier ${ }^{(29)}$. O alongamento realizado agudamente pode influenciar positivamente a economia de movimento, porém ainda assim a força e a velocidade de contração são diminuídas, possivelmente pelo fato de ocorrer um maior prejuízo à fibra muscular ${ }^{(30)}$. Esses resultados vão de encontro com a hipótese de que a diminuição da força muscular após um protocolo agudo de alongamento seja causada pela diminuição da ativação das unidades motoras ${ }^{(28)}$

\section{CONCLUSÃO}

São contraditórias as informações envolvendo alongamento e treinamento de força, sendo os diferentes protocolos utilizados nos experimentos uma das possíveis causas para a contradição entre os resultados. É importante salientar ainda que alguns atletas alongam antes de uma sessão de força com o intuito de diminuir os riscos de lesões decorrentes da sessão de treinamento, porém estudos demonstram que o alongamento não necessariamente diminui os riscos de lesões provenientes do treinamento ${ }^{(31,32)}$.

\section{REFERÊNCIAS BIBLIOGRÁFICAS}

1. Fisher MM. The effect of resistance exercise on recovery blood pressure in normotensive and borderline hypertensive women. J. Strength Cond. Res 2001; 15:210-6.

2. Farrell PA, Fedele MJ, Hernandez J, Fluckey JD, Miller III JL, et al. Hypertrophy of skeletal muscle in diabetic rats in response to chronic resistance exercise. J Appl Physiol 1999; 87:1075-82. 
3. Burleson JR, O'byant HS, Stone MH, Collins MA, Triplettmcbride $\mathrm{T}$. Effect of weight training exercise and treadmill exercise on post-exercise oxygen consumption. Med Sci Sports Exerc 1998; 30:518-522.

4. Elsangedy, HM, Krinski, K, Jabor, IAS. Efeito do exercício resistido em mulheres idosas portadoras de osteoporose. Lecturas Educación Física y Deportes 2006; 100:1-7.

5. Azevedo P.H.S.M, Demampra TH, Oliveira GP, Baldissera V, Burger-Mendonça M, Marques AT, et al et al. Efeito de 4 semanas de treinamento resistido de alta intensidade e baixo volume na força máxima, endurance muscular e composição corporal de mulheres moderadamente treinadas. Brazilian Journal of Biomotricity 2007; 1:76-85.

6. Lamas, L.; Drezner, R.; Tricoli, V.; Ugrinowitsch, $C$. Efeito de dois métodos de treinamento no desenvolvimento da força máxima e da potência muscular de membros inferiores. Rev Bras de Educação Física e Esp 2008. 22: 235-245.

7. Felício, JM; Barquilha, G. Comparação entre membros superiores e inferiores perante diferentes intervalos de descanso em exercícios resistidos. Lecturas Educación Física y Deportes 2009: 135:01-01.

8. Bishop D. Warm up II: performance changes following active warm up and how to structure the warm up. Sports Med 2003: 33:483-98.

9. Coutinho EL, Gomes ARS, França CN, Oishi J, Salvini TF. Effect of passive stretching on the immobilized soleus muscle fiber morphology. Braz. J. Med. Biol. Res 2004; 37:1853-1861.

10. Gomes ARS, Cornachione A, Salvini TF, MattielloSverzut AC. Morphological effects of two protocols of passive stretch over the immobilized rat soleus muscle. J. Anat 2007; 210:328-335.

11. Tricoli V, Paulo AC. Efeito agudo dos exercícios de alongamento sobre o desempenho de força máxima. Revista Brasileira de Atividade Física e Saúde 2002; 7:6-12.
12. Young W.B, Behm D.G. Effects of running, static stretching and practice jumps on explosive force production and jumping performance. J Sports Med Phys Fitness 2003; 43:27-37.

13. Shier L. Does stretching improve performance? A systematic and critical review of the literature. Clin J Sports Med 2004;14:267-73.

14. Ribeiro FM, Oliveira $F$, Jacinto $L$, Santoro $T$, Lemos A, Simão R. Influência aguda do alongamento passivo e do aquecimento específico na capacidade de desenvolver carga máxima no teste de 10RM. Fit Perf J 2007; 6: 5-9.

15. Francisco RV, Lima AJG, Cosenza PI. Efeito agudo do alongamento e de ambos no número máximo de repetições realizadas com $80 \%$ de 1 RM no supino. In: XXVII Simpósio Internacional de Ciências do Esporte. Rev Bras Ciência e Mov 2004;12:223.

16. Arruda, FLB; Faria, LB ; Silva, V ; Senna, GW ; Simão, R; Novaes, J; Maior, AS . A influência do alongamento no rendimento do treinamento de força. Trein Desportivo 2006; 07: 01-05.

17. Endlich PW, Farina GR, Dambroz C, Goncalves WLS, Moyses MR, Mill JG, Abreu, GR. Efeitos agudos do alongamento estático no desempenho da forca dinâmica em homens jovens. Rev Bras Med Esp 2009; 5: 200-203.

18. Costa EC, Santos CM, Prestes J, Silva JB, Knackfuss MI. Efeito agudo do alongamento estático no desempenho de força de atletas de jiu-jitsu no supino horizontal. Fit Perf J 2009; 8: 212-7.

19. Souza, ACR.; Bastos, CLB.; Portal, MND.; Salles, BK.; Gomes, TM.; Novaes, JS. Efeito agudo do intervalo passivo e do alongamento no desempenho de séries múltiplas. Rev Bras Cineantropom Desempenho Hum 2009, 11: 435-443.

20. Yamaguchi, T; Ishii, K. Effects of static stretching for 30 seconds and dynamic stretching on leg extension power. J. Strength Cond. Res. 2005; 19:677-683.

21. Kokkonen J, Nelson AG, Cornwell A. Acute muscle stretching inhibits maximal strength performance. Res Q Exerc Sport. 1998;69::411-5. 
22. Grego Neto, A.; Manffra, EF. Influência do volume de alongamento estático dos músculos isquiotibiais nas variáveis isocinéticas. Rev Bras Med Esp. 2009; 15:104-109.

23. Benjamin J.P; Lamp, S. Understanting sports massage. $2^{\circ}$ ed. Champaign, Human Kinets, 1996. IN: ACHOUR JUNIOR, A. Anatomia e fisiologia para exercícios de alongamento. Manole, 2002.

24. Behm DG, Button DC, Butt JC. . Factors affecting force loss with prolonged stretching. Can J Appl Physiol $2001 ; .26: 262-72$.

25. Nelson AG, Guillory IK, Cornwell C, Kokkonen J.. Inhibition of maximal voluntary isokinetic torque production following stretching is velocity-specific. J. Strength Cond. Res. 2001; 15:241-6.

26. Kubo k, Kanehisa H, Kawakami Y, Fukunaga T. Influence of static stretching on viscoelastic properties of human tendon structures in vivo. J Appl Physiol 2001;90:520-7, 2001.

27. Wilson GJ, Murphy AJ, Pryor JF. Musculotendinous stiffness: its relationship to eccentric, isometric, and concentric performance. J Appl Physiol 1994; 76:.271419.

28. Marek SM, Cramer JT, Fincher AL, Massey LL, Dangelmaier SM, Purkayastha S, et al. Acute Effects os Static and Proprioceptive Neuromuscular Facilitation Stretching on Mucle Strength and Power Output. J Athl Train 2005; 40:94-103
29. Shrier I. Stretching before exercise does nor reduce the risk of local muscle injury: a critical review of the clinical and basic science literature. Clin J Sport Méd 1999; 9:221-227.

30. Magnusson SP, Simonsen EB, Aagaard P, Sorensen $\mathrm{H}$, Kjaer M. A mechanism for altered flexibility in human skeletal muscle. Am J Sports Med 1996; 24:622-628.

31. Pope RP, Herbert RD, Kirwan M, Grahan BJ. $A$ randomized trial of preeexercise stretching for prevention of lower-limb injury. Med Sci Sports Exerc 2000; 32:271-277.

32. Shrier I. Stretching before exercise does nor reduce the risk of local muscle injury: a critical review of the clinical and basic science literature. Clin J Sport Med 2000; 9:221-227.

Endereço para correspondência

Universidade Cruzeiro do Sul - Instituto de Atividade

Física e Esporte

Rua Galvão Bueno, 868 - 13 Bloco B

Liberdade - São Paulo - SP

Cep 01506-000

Email: gustavo_barquilha@hotmail.com 